\title{
Shape Memory Electrospun Nonwovens Based on Crosslinked Poly(E-Caprolactone) for Multifunctional Biological Applications
}

\author{
Nicoletta Inverardi ${ }^{a}$, Stefano Pandini ${ }^{a}$, Silvia Agnellia, Paola S. Ginestra ${ }^{a}$, Rosalba \\ M. Ferraro ${ }^{\mathrm{b}}$, Sarah Tonello ${ }^{\mathrm{c}}$, Mariagrazia Marziano ${ }^{\mathrm{c}, \mathrm{d}}$, Mauro Serpelloni ${ }^{\mathrm{c}}$, Nicola F. \\ Lopomo $^{\mathrm{c}}$, Andrea Merlettini ${ }^{\mathrm{e}}$, Chiara Gualandi ${ }^{\mathrm{e}}$, Maria Letizia Focarete ${ }^{\mathrm{e}}$, Massimo \\ Messori $^{\mathrm{f}}$, Maurizio Toselli ${ }^{\mathrm{g}}$
${ }^{a}$ Department of Mechanical and Industrial Engineering, University of Brescia, via Branze 38, 25123, Brescia, Italy
${ }^{b}$ Department of Molecular and Translational Medicine, "Angelo Nocivelli" Institute of Molecular Medicine, University of Brescia, pl.e Spedali Civili 1, 25123, Brescia, Italy
${ }^{c}$ Department of Information Engineering, University of Brescia, via Branze 38, 25123, Brescia, Italy
${ }^{e}$ Department of Chemistry “G. Ciamician”, University of Bologna, via Selmi 2, 40126, Bologna, Italy
${ }^{f}$ Department of Engineering "E. Ferrari”, University of Modena and Reggio Emilia, via P. Vivarelli 10/1, 41125, Modena, Italy
${ }^{g}$ Department of Industrial Chemistry "Toso Montanari”, University of Bologna, viale Risorgimento 4, 40136, Bologna, Italy \\ ${ }^{d}$ Department of Molecular and Translational Medicine, University of Brescia, viale Europa 11, 25123, Brescia, Italy
}

\begin{abstract}
In this work we have explored the capabilities of an electrospun mat, realized in crosslinked poly( $\varepsilon$ caprolactone), to be employed as a scaffold for specific biological applications. Its shape memory behavior was here exploited to easily control fiber orientation and to guide cellular alignment. Randomly oriented mats were transformed in a stable shape with various degrees of fiber alignment by simply varying the maximum strain applied throughout proper thermo-mechanical cycles. The effect of fiber alignment was investigated both on mechanical properties and on cell culture, through biological characterization done by using Neural Stem Cells derived from human iPSCs (induced Pluripotent Stem Cells). Further, thanks to the thermal stability of the microfibrous structure, it was possible to transfer, through inkjet printing on the electrospun, a biocompatible sensing element, which successfully allowed to monitor cell adhesion (L6 Myoblast) in an innovative way under dynamic conditions.
\end{abstract}

Keywords: electrospinning; shape memory polymers; scaffold; neural stem cells; sensor PACS: 87.85.J-; 62.20.fg; 82.47.Rs

\section{INTRODUCTION}

Electrospun nonwovens based on biocompatible polymers are frequently employed as scaffolds for tissue engineering thanks to their interconnected porosity and large surface-to-volume ratio. Recently, the possibility to realize scaffolds based on shape memory polymers is investigated in order to obtain systems able to change both their shape and their microstructure when heated at specific temperatures. This behavior may provide additional features, such as the possibility of self-deployment in minimally invasive surgery applications [1], or the ability to control cellular growth thanks to fiber alignment [2]. In fact, while most of nonwovens are made by randomly oriented nano-/micrometric fibers, an aligned fibrous microstructure may be important to fulfil the strict requirements of tissue engineering, such as, for instance to better mimic the morphology of the extracellular matrix.

In this work some features related to the shape memory behavior of a randomly oriented nonwoven based on crosslinked poly( $\varepsilon$-caprolactone) were exploited to realize functional scaffolds with specific performances. First, in 
addition to traditional way to obtain fiber alignment by modifying the electrospinning set-up [3], shape memory capabilities turned helpful, thanks to the possibility to temporarily fix a given microstructure through deformation. This effect was explored through the application of thermo-mechanical cycles to achieve various degrees of fiber alignment and the change in mechanical properties, as well as the possibility to orient cell growth, was measured. Further, thanks to the stability of the fibrous microstructure above $\mathrm{T}_{\mathrm{m}}$, it was possible to print, by a thermal treatment at relatively high temperature, a sensing element on the scaffold surface to indirectly monitor cell adhesion, following a recent and promising research field in Electrochemical Cell-based Impedance Spectroscopy (ECIS) [4].

\section{EXPERIMENTAL}

Electrospun mats of crosslinked poly( $\varepsilon$-caprolactone) (PCL) were produced starting from triethoxysilaneterminated PCL precursors through a process that combines electrospinning and sol-gel reaction, following a three steps protocol, described in details in [5], which consists in: (1) partial crosslinking of PCL precursors; (2) electrospinning of the partial reacted solution to obtain bead-free fibers; (3) post-crosslinking treatment in acid environment. The obtained nonwoven system is a $50 \mu \mathrm{m}$ thick mat, formed by randomly oriented fibers, with an average diameter of about $2 \mu \mathrm{m}$.

Randomly oriented mats were subjected to a thermo-mechanical cycle in order to provide various degrees of alignment of the fibrous microstructure. The cycles were carried out on rectangular strips (average gauge length: 10 $\mathrm{mm}$, average width: $0.5 \mathrm{~mm}$ ) in a dynamic mechanical analyzer (DMA Q800; TA Instruments) under tensile configuration, as follows: (1) heating at $80{ }^{\circ} \mathrm{C}$ (i.e. well above melting temperature, $\mathrm{T}_{\mathrm{m}}=55^{\circ} \mathrm{C}$ ); (2) deformation under load control condition (loading rate: $0.01 \mathrm{~N} / \mathrm{min}$ ) up to various strain levels $\left(\varepsilon_{\text {appl }}=25 \% ; 50 \% ; 100 \% ; 185 \%\right)$; (3) cooling to room temperature (i.e. below crystallization temperature $\mathrm{T}_{\mathrm{c}}=31^{\circ} \mathrm{C}$ ) under fixed strain. Random and variously oriented mats were subjected to mechanical tests under tensile condition. The tests were carried out at room temperature by means of the dynamic-mechanical analyzer, under load control with a loading rate equal to 0.2 $\mathrm{N} / \mathrm{min}$. In addition cyclic loading-unloading tests were performed in order to identify the best operative conditions to fulfil the requirements of a dynamic cell culture, i.e. a maximum strain amplitude of $10 \%$ and to avoid buckling of the scaffold during unloading. Moreover, the crosslinked structure of the polymer permits to heat the mat above $\mathrm{T}_{\mathrm{m}}$ region without losing the fiber morphology. This feature allowed to print, through the application of ink-jet printing technique and a subsequent heat treatment at $110^{\circ} \mathrm{C}$, a cytocompatible sensing element on the mat, in order to use it as a scaffold capable of monitoring cell adhesion, as recently done and reported [4].

Cell culture tests were performed to investigate the effects of fiber arrangement on the cellular orientation and to validate the possibility to use PCL-based inkjet printed sensors for cell monitoring.

Regarding the first application, the randomly oriented mat and the most aligned one $\left(\varepsilon_{\text {appl }}=185 \%\right)$ were used as scaffolds for Neural Stem Cells (NSCs) derived from human induced Pluripotent Stem Cells (iPSCs). Before cell seeding, all the scaffolds, cut in $1 \mathrm{~cm}^{2}$ strips and placed in a 24-well, were washed with PBS (Phosphate Buffered Saline) and underwent a sterilization protocol composed by a treatment with $\mathrm{NaOH} 1 \mathrm{M}$ for 1 hour, a sterilization with $70 \%$ ethanol followed by a step under UV light for 30 minutes. Finally scaffolds were coated with Matrigel matrix to improve cell adhesion. iPSCs-derived NSCs were dissociated with Accutase, plated at a cellular density of 80000 cells $/ \mathrm{cm}^{2}$ and maintained in a proper growth medium. Seeded scaffolds were incubated at $37{ }^{\circ} \mathrm{C}$ with $5 \%$ $\mathrm{CO}_{2}$. In order to evaluate cell adhesion and orientation, after 7 days, cells were fixed using the Fix\&Perm Sample Kit for 30 minutes (15 minutes fixation and 15 minutes permeabilization), incubated with blocking solution for 45 minutes, and stained with Phalloidin that marks the cytoskeletal component of the cells. Cell nuclei were then counterstained with DAPI (4'-6-diamidino-2- phenylindole) for 5 minutes to highlight the cellular nuclei of the living cells. The samples were mounted onto glass slides and observed with an inverted fluorescent microscope. Cell orientation distribution was measured by using Image $\mathrm{J}$ software.

In the case of cell monitoring application, impedance based measurements were performed - both in static and dynamic (i.e. under cyclic loading-unloading of the scaffold) conditions - before seeding and after 24 hours, in order to correlate impedance changes with cell adhesion. Measurements were repeated with three different cell concentrations $\left(15000,30000\right.$ and $\left.60000 \mathrm{cells} / \mathrm{cm}^{2}\right)$, in order to evaluate the sensitivity of the technique.

\section{RESULTS}

As discussed in [5], both dynamic-mechanical analysis and swelling tests revealed an effectively crosslinked structure for the polymer constituting the mat fibers. This allows to obtain good shape memory response, so that mats are capable to fully fix and recover even high deformations (above 100\%). In order to change the 
microstructure towards increased levels of fiber alignment, electrospun mats were deformed above $\mathrm{T}_{\mathrm{m}}$ up to various strain levels and set in their deformed shape by cooling under fixed strain to room temperature. SEM (Scanning Electron Microscopy) images reported in Fig. 1 highlight the effect of the applied strain [5]. For the applied strain below 50\% the overall fiber arrangement remains practically random, while higher strains determine a progressive increase of fiber alignment in the direction of applied strain. In particular, for the maximum applied strain (185\%) the fiber alignment appears significant: more than $70 \%$ of considered fiber segments are aligned in a direction within $0^{\circ} \pm 15^{\circ}$, whereas the pristine randomly oriented mat does not show any preferred direction of fiber alignment. A further confirmation of the strain effect on fiber alignment may be obtained, indirectly, by taking into account the change in the mechanical behavior of the deformed mat. Tensile tests were carried out on the deformed systems, and results are reported in Fig. 1 in terms of nominal stress vs. nominal strain curves (the nominal strain being determined by considering the specimens fixed at various $\varepsilon_{\text {appl }}$ as they were in their undeformed state). The stretched mats show an effective increase in stiffness only from applied strain of $50 \%$ and an overall change in the initial modulus from about $20 \mathrm{MPa}$ (undeformed mat; $\left.\varepsilon_{\mathrm{appl}}=25 \%\right)$ to $45 \mathrm{MPa}\left(\varepsilon_{\mathrm{appl}}=185 \%\right)$.

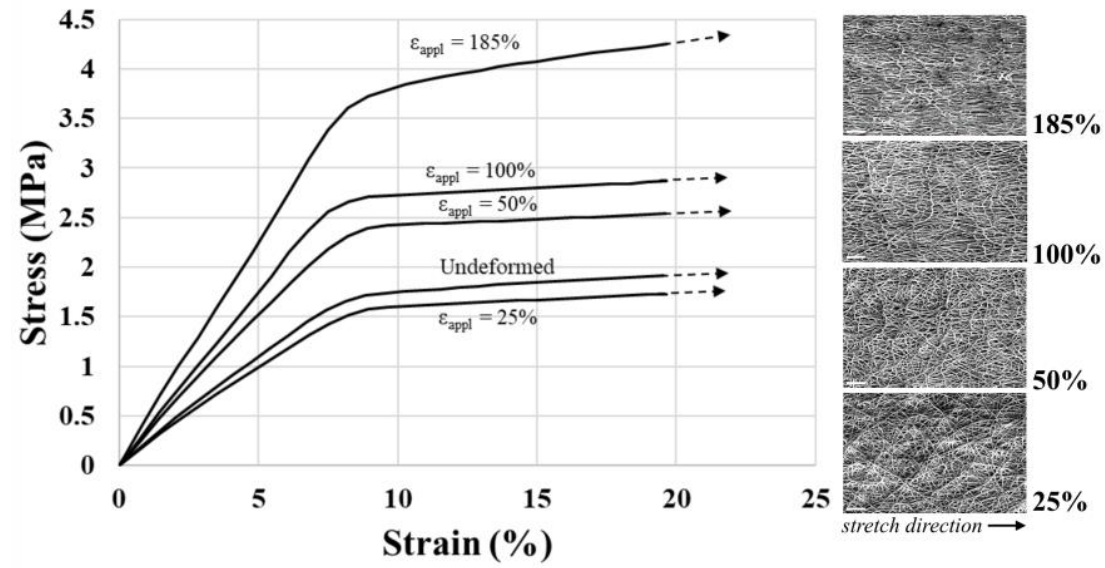

FIGURE 1. Stress-strain curves for randomly oriented and variously aligned electrospun mats, and SEM images of the mats fixed at various deformation levels (scale bars: $40 \mu \mathrm{m}$ ).

The ability to sustain cell adhesion, first investigated by seeding balb/3T3 clone A31 mouse embryo fibroblasts on the undeformed mat, revealed a lack of cytotoxicity, allowing to consider these systems as potential scaffolds for tissue engineering [6]. To investigate the possibility to control cellular alignment, biological tests were carried out on both randomly oriented and highly aligned mats, seeding them with iPSCs-derived NSCs. After 7 days, cells were stained to test the cellular adhesion and survival on randomly oriented and highly aligned scaffolds. The biological tests show cytocompatibility for both fiber arrangements. Further, a remarkable difference in cell culture can be detected based on the morphology of the scaffold, as seen in Fig. 2. In fact, the randomly oriented mat supports cell growth in all directions (Fig. 2a), whereas cells on the highly aligned mat seem to follow fiber direction (around $12^{\circ}$ in Fig. 2b) with a mean data of $1.43^{\circ}$ for cell cytoskeleton alignment, as highlighted by statistical analysis reported in Fig. 2c.
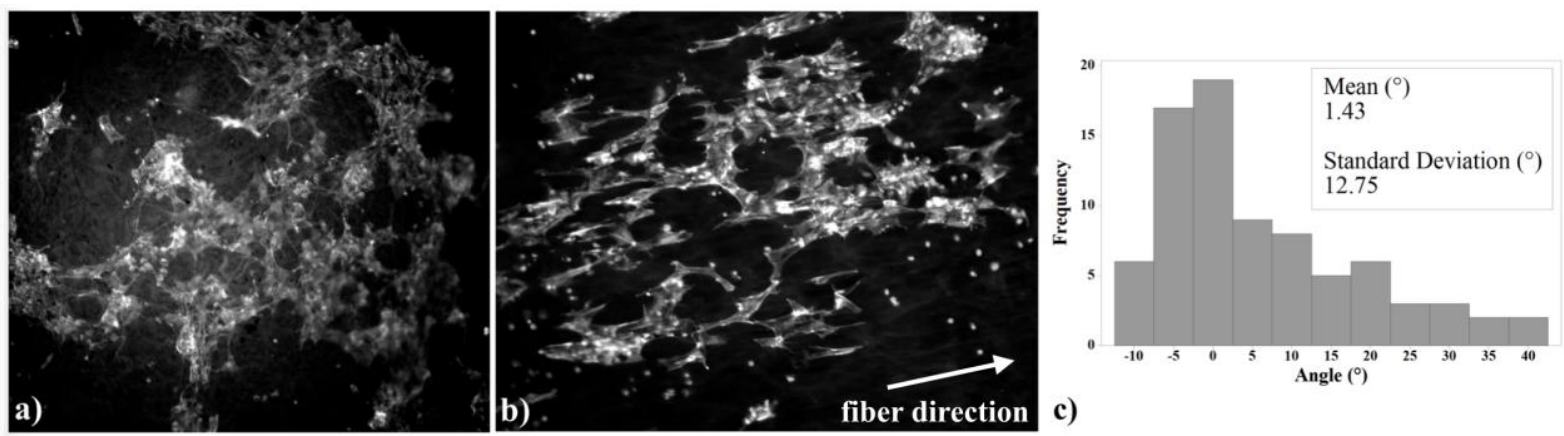

FIGURE 2. Fluorescent microscopy of iPSCs-derived NSCs seeded on the randomly oriented mat (a) and on the highly aligned mat $\left(\varepsilon_{\mathrm{appl}}=185 \%\right.$, fiber direction $\left.\approx 12^{\circ}\right)(\mathrm{b}), 10 \mathrm{x}$ magnification. $\left.\mathrm{c}\right)$ Distribution of cytoskeleton alignment on the aligned mat. 
Furthermore, cell nuclei, from their characteristic round shape, become more oval on the aligned mat, suggesting the alignment of the entire cell body. These results encourage the chance to use aligned scaffolds to promote cell differentiation in neurons, characterized by long and thin cytoskeletal protrusions.

The opportunity to monitor cell adhesion through an ink-jet printed biocompatible sensor on randomly oriented scaffolds was exploited with a more common cell line (L6 Myoblast), giving promising results in both static and dynamic conditions. The latter ones concern the cyclic deformation of the scaffold, optimized for a constant strain amplitude of $10 \%$ without any negative stress, and therefore without buckling effects, as shown in Fig. 3a for both random and highly aligned mats; the stable mechanical stimulation is ensured by a previous training protocol on the scaffold (50 cycles between 2,5\% and 5\% strain; 50 cycles between 5\% and $10 \%$ strain; 100 cycles between $10 \%$ and $20 \%$ strain) and by applying on the trained scaffold loading-unloading cycles between $7 \%$ and $17 \%$. Thus the mechanical stimulus could be helpful in promoting cell maturation. As can be seen in Fig. $3 \mathrm{~b}$, the sensor is able to measure impedance magnitude variation before and after cell seeding (i.e. $\Delta$ Magnitude) with an increasing sensitivity as the seeded cell density increases. The increase in impedance magnitude variation with cell concentration is a common feature throughout the frequency range explored (from $400 \mathrm{~Hz}$ to $1000 \mathrm{kHz}$ ) and it is pointed out in Fig. $3 \mathrm{~b}$ for the two frequencies $(4 \mathrm{kHz}$ and $100 \mathrm{kHz})$ known to be interesting for impedance based cell culture monitoring.
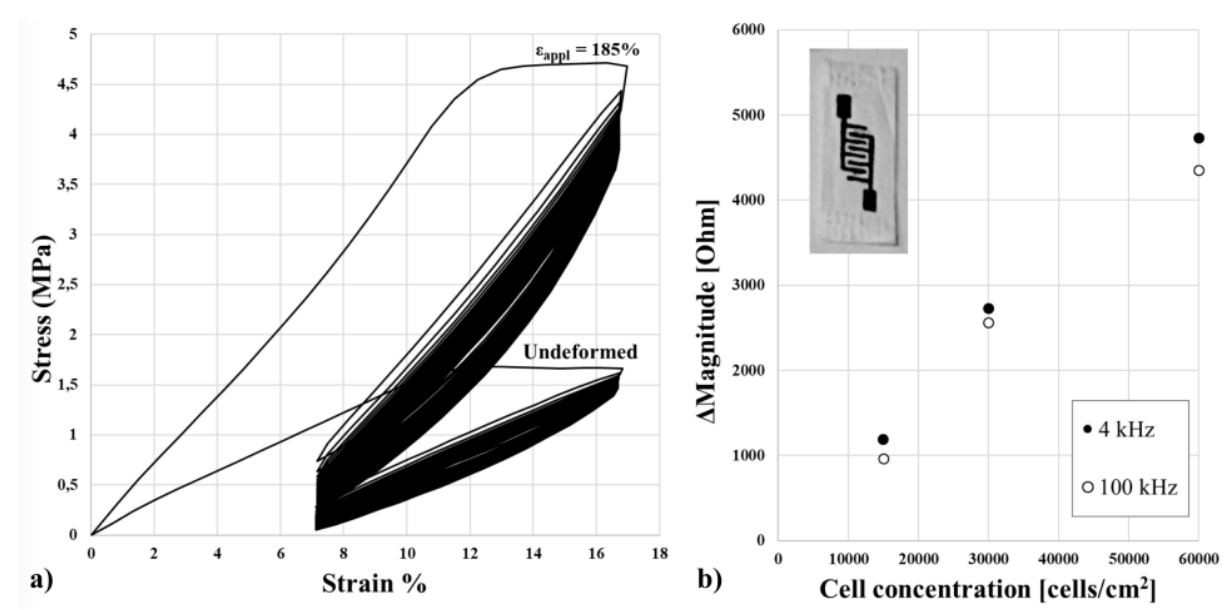

FIGURE 3. a) Cyclic behavior in terms of stress-strain curves for the randomly oriented mat and the highly aligned one. b) Impedance magnitude variation (before and after L6 cells seeding) vs. three different cell concentrations tested (15000, 30000, 60000 cells $/ \mathrm{cm}^{2}$ )

The possibility to easily obtain highly aligned mats (after $185 \%$ applied deformation), which can sustain cyclic deformations without buckling, could be exploited in the future in combination with the printed sensor in order to guide, through fiber alignment, and simultaneously control, through the impedance sensor, the cell differentiation process.

\section{REFERENCES}

1. M. Bao, X. Lou, Q. Zhou, W. Dong, H. Yuan, Y. Zhang, ACS Appl. Mater. Interfaces, 6, 2611-2621 (2014).

2. J. Wang, A. Quach, M.E. Brasch, C.E. Turner, J.H. Henderson, Biomaterials 140, 150-161 (2017).

3. R. Murugan, S. Ramakrishna, Tissue Engineering 13 (8), 1845-1866 (2007).

4. M. Marziano, S. Tonello, M. Serzanti, M. Borghetti, N.F. Lopomo, M. Serpelloni, S. Pandini, A. Merlettini, C. Gualandi, M.L Focarete, M. Messori, M. Toselli, D. Uberti, M. Memo, P. Dell'Era, E. Sardini, “Carbon on poly(e-caprolactone) (PCL) inkjet printed sensor for monitoring cell cultures of myoblasts", EMBEC \& NBC, 783-786 (2017).

5. A. Merlettini, S. Pandini, S. Agnelli, C. Gualandi, K. Paderni, M. Messori, M. Toselli, M. L. Focarete, RSC Adv. 6, $43964-$ 43974 (2016).

6. S. Pandini, S. Agnelli, A. Merlettini, F. Chiellini, C. Gualandi, K. Paderni, M.L. Focarete, M. Messori, M. Toselli, Macromol. Mater. Eng. 302 (8), 1600519 (2017). 